\title{
Structural and light-emission modification in chemically-etched porous silicon
}

\author{
D. Navarro-Urrios ${ }^{*}{ }^{1,2}$, C. Pérez-Padrón ${ }^{1}$, E. Lorenzo ${ }^{1}$, N. E. Capuj ${ }^{1}$, Z. Gaburro ${ }^{2}$, \\ C. J. Oton ${ }^{1,2}$, and L. Pavesi ${ }^{2}$ \\ ${ }^{1}$ Departamento de Física Básica, University of La Laguna, Avda. Astrofísico Fco. Sánchez, La Laguna, \\ 38071 Spain \\ 2 INFM and Dipartimento di Física, University of Trento, Via Sommarive 14, Povo, Trento, 38050 Italy
}

Received 12 July 2004, revised 24 October 2004, accepted 27 January 2005

Published online 25 May 2005

PACS 42.79.Ci, 42.82.-m, 61.43.Gt, 68.65.Ac, 81.05.Rm, 82.45.Vp

After electrochemical etching, we have made a study of the effects generated on $\mathrm{p}^{+}$-type porous silicon layers when they are left in presence of the electrolyte for different post-etching times. Using an interferometric technique, we have monitored the change of its porosity during the post-etch process due to a chemical dissolution mechanism. These data are complemented with a study of photoluminescence and transmission electron microscopy measurements for different post-etching times.

() 2005 WILEY-VCH Verlag GmbH \& Co. KGaA, Weinheim

\section{Introduction}

The nanostructured configuration of porous silicon (PS) has fascinating properties which results mainly from quantum confinement of excited electron-hole pairs. Light emission under optical and electrical pumping in the visible and at room temperature has been observed in the last years [1,2]. Other applications of PS as a passive material have been demonstrated [3-6]. For instance, the modulation of the electrochemical current with time during the growth allows an easy realization of multilayer structures that can behave as distributed Bragg reflectors (DBRs) [7, 8], microcavities [9], waveguides [10], etc.

The optical properties of PS are determined by both the complex network of nanometric air pores and by the silicon skeleton. Usual treatment assumes an effective medium approximation where the refractive index of PS is the results of some weighted average of the contributions of the air pores and of the crystalline skeleton. Therefore the optical properties of PS are strongly dependent on the details of its structure. It has been demonstrated that chemical etching of PS in HF enlarges the diameter of the pores and thus alters its properties. Emission spectra of these post-etched layers were blue shifted with respect to those of the original PS layers which supports an hypothesis of a reduction of the sizes of the silicon skeleton which in turn increases the quantum confinement of carriers and, hence, the energy of the emission [1,11].

Using in-situ laser beam interference, the optical path of the PS layer $(n l)$, where $n$ is the refractive index and $l$ the thickness) can be monitored during anodization [12]. Even two different beams with different incident angles can be analyzed simultaneously to obtain information about the porosity and etch rate [13]. This kind of in-situ measurements have the advantage of allowing a continuous monitoring of the optical properties of the PS layer during the anodization. In this work we make use of this insitu interferometric technique to study the etching in HF of a PS layer after the anodization process. We complement these data with photoluminescence (PL) measurements, and a transmission electron microscopy (TEM) characterization made in self-supported samples.

\footnotetext{
Corresponding author: e-mail: navarro@science.unitn.it
} 


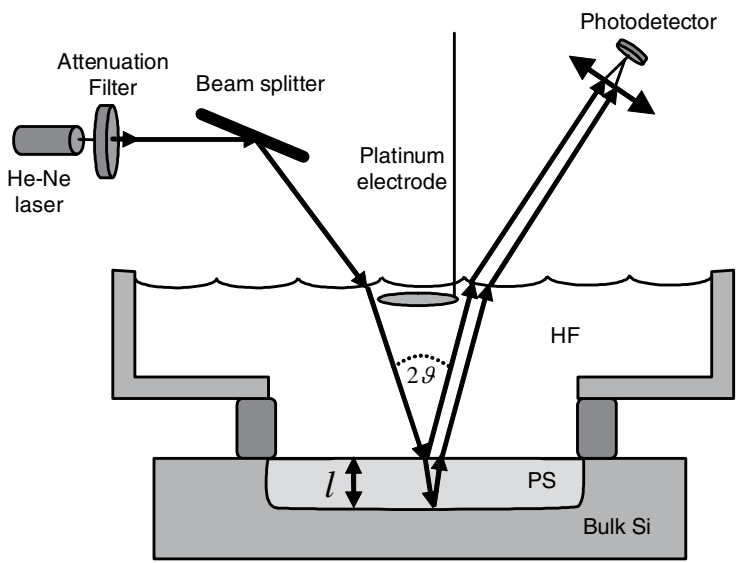

2 Experimental set-up and theoretical background

Figure 1 sketches the experimental setup. The sample immersed in the electrolyte is illuminated by an extremely weak laser beam at an angle $\vartheta$. The reflectance of this beam is measured as a function of time during the electrochemical etching. The intensity of the reflected beam is mainly the result of the interference between the two beams reflected at the electrolyte/top PS and bottom PS/bulk silicon interfaces. In order to analyze the interference, a ray equation has to be solved. Let us assume that a layer is being etched with a constant current density, i. e. we assume that the refractive index $n_{\mathrm{PS}}$ of PS remains constant. It can be shown that the optical path difference $D$ between the two different beams is:

$$
D=2 l \sqrt{n_{\mathrm{PS}}^{2}-n_{\mathrm{HF}}^{2} \sin ^{2}(\vartheta)}
$$

where $l$ is the PS thickness, $n_{\mathrm{HF}}$ is the refractive index of the electrolyte, and $\vartheta$ is the incidence angle of the beam. Similarly to what occurs during the etching stage, an oscillating signal in time can be measured during the post-etching. If this occurs, it means that during the post-etching treatment the optical path of the PS layer is changing. As large variations of $l$ are unlikely, most of the changes of $D$ are due to the reduction of the density of PS caused by the chemical etching due to HF. The frequency of the reflectance during the post-etching process $v_{\mathrm{pe}}(t)$, assuming also small incident angles $\left(\vartheta<10^{\circ}\right)$ becomes:

$$
\nu_{\mathrm{pe}}(t) \cong \frac{2 l}{\lambda_{0}} \frac{\mathrm{d} n_{\mathrm{PS}}}{\mathrm{d} t} .
$$

Equation (2) relates the oscillating reflectance frequency with the variation in the PS refractive index. The PS samples were fabricated from $\mathrm{p}^{+}$-type boron-doped $\mathrm{Si}$ substrates of low resistivity $(0.01 \Omega \mathrm{cm})$. The electrochemical etching was performed at room temperature in electrolyte containing 1:3 mixture of $48 \% \mathrm{HF}$ and ethanol. We performed the electrochemical etching in the dark applying a constant current density of $40 \mathrm{~mA} / \mathrm{cm}^{2}$ on an area of $1 \mathrm{~cm}^{2}$. The HP 6612C current source was controlled by a computer.

Self-supported samples suitable for TEM measurements were made by applying anodic current pulse $\sim 0.4 \mathrm{~A} / \mathrm{cm}^{2}$ for $0.5 \mathrm{~s}$ to detach the structure from the substrate. Once the electrochemical etching was ended, the PS layer was left in the electrolyte in dark, and the chemical post-etching was initiated. Several post-etching duration times were used. For photoluminescence and TEM measurements the PS layers were taken out from the HF dissolution, rinsed in ethanol and dried in ambient air.

The optical set up of the experiment is shown in Fig. 1. The in situ monitoring of the optical path change was performed using a $632.8 \mathrm{~nm} \mathrm{He}-\mathrm{Ne}$ laser with a power of $1 \mu \mathrm{W}$. This low power level assures no effect on the electrochemical etching and post-etching due to the light beam. The incidence angle of the laser beam on the sample was $8^{\circ}$ and the spot size was $\sim 2 \mathrm{~mm}$. The reflected beam was 


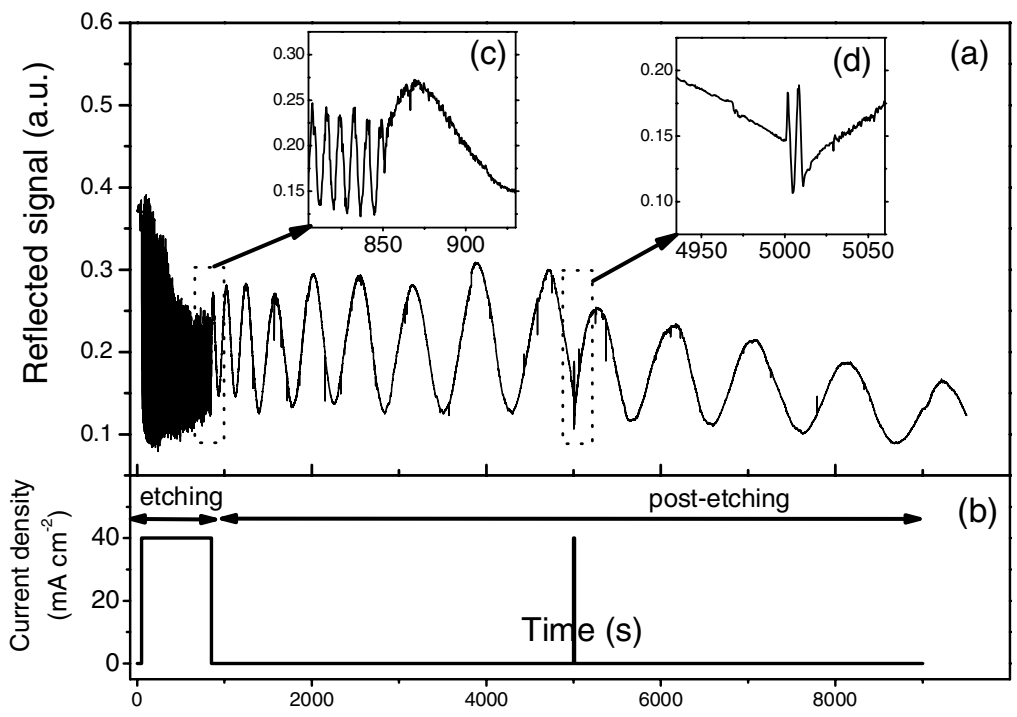

Fig. 2 (a) Reflected signal during the anodization and the post-etching processes. (b) Electrochemical current density applied versus time ( $800 \mathrm{~s}$ of anodization time and a short pulse after $4150 \mathrm{~s}$ of post-etching). (c) and (d) Blowup of the reflected signal near the current changes. The frequency and the phase sign changes can be clearly observed.

focused onto a silicon photodiode in order to improve the stability of the signal. The current signal of the photodiode was amplified and acquired with a computer. The photoluminescence (PL) experiments were performed pumping with all the lines of an Ar CW laser, focalizing a $100 \mathrm{~mW}$ beam on the sample.

\section{Results}

We have made samples with different electrochemical etching times $(400,800,1200$ and $1600 \mathrm{~s})$ and we analyzed the post etching for much longer times (up to 18000 s). In Fig. 2a we show the oscillating intensity of the reflected beam for one sample with $800 \mathrm{~s}$ of etching and in Fig. $2 \mathrm{~b}$ the corresponding current density as a function of time. The fast oscillations during the etching time are due to the fast increase of optical path [13] (Fig. 2c). Contrary to expectations, once the current is stopped, instead of a constant signal we observed reflectance oscillations with a sudden decrease of the frequency. This indicates that some kind of etching is occurring even when there is no current. It is also worth noting in Fig. $2 \mathrm{c}$ that the phase of the oscillating reflectance signal changes sign. Since during the electrochemical etching the optical path of the layer grows, a change of phase when the current is stopped indicates that the optical path starts to decrease. This can be explained by a decrease of the refractive index of the PS layer due to the chemical etching of the silicon. It is also interesting the fact that the frequency of the reflectance oscillations decreases during the post-etching process. One could attribute the low frequency observed during the post-etching to some slow variation of the electrolyte composition, due to the out-diffusion of chemical components when the current is stopped (e.g. $\mathrm{F}^{-}$ions). This diffusion could change the refractive index producing a change in the optical path of the layer. However, we can discard this hypothesis because, after $4150 \mathrm{~s}$ of chemical etching, i.e. at time $5000 \mathrm{~s}$, a $10 \mathrm{~s}$ short current pulse was applied to increase the PS layer thickness and to recover the electrolyte composition within the PS layer (shown in Fig. 2d). The frequency of the signal clearly retrieves the value it had before the current step. This fact demonstrates that the slow oscillations during the post-etching are not a transient occurred after any current stop, but a consequence of a irreversible change in the PS structure The decreasing frequency of the signal during post-etching means that the etch velocity of PS by the electrolyte decreases with time. 

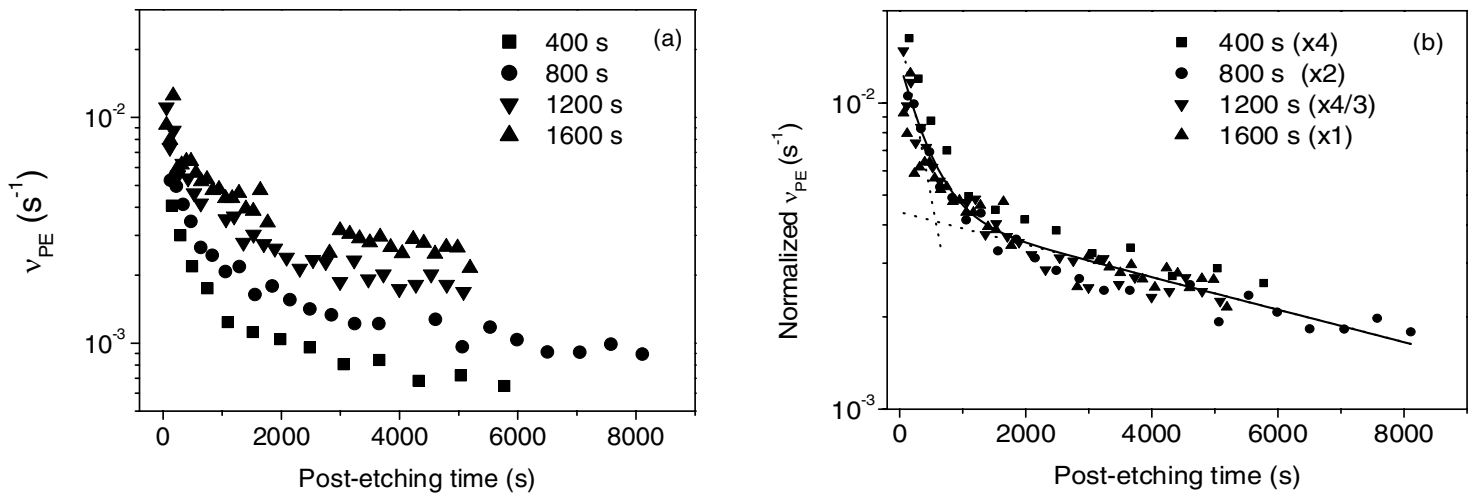

Fig. 3 (a) Frequency of the reflected oscillating signal during etching of PS samples formed at the same current but with changing electrochemical etching times. (b) Normalized frequency vs the post-etching time (normalization is done by multiplying the frequency by $1600 \mathrm{~s}$ and dividing by the corresponding electrochemical etching time). Scaling factors are shown within parenthesis. The symbols refer to the same sample as in (a). The line is a two exponential fit to the data.

Figure 3 a shows the frequency of the signal versus the post-etching time for four different PS samples. In Fig. $3 \mathrm{~b}$ the thickness normalized frequencies versus the post-etching time are reported for the various PS samples. All the data points align that supports the theoretical model we used (the refractive index of the already etched part remains almost constant during the anodization) and implies that the rate of refractive index variation is the same for the different PS layer thickness. The data can be fitted with a double exponential with $t_{1}=400 \mathrm{~s}$ and $t_{2}=8200 \mathrm{~s}$ (lines in Fig. 3b).

Figure 4 shows the PL measurements for various PS samples obtained with $800 \mathrm{~s}$ of electrochemical etching but with different post-etching duration. In the first minutes of the post-etching treatments (Fig. 4a), a slight luminescence line shape change is observed where the relative intensity of the short wavelength $(550-700 \mathrm{~nm})$ emission increases until a maximum is reached at $1800 \mathrm{~s}$ of post-etching time. For longer times, this short wavelength contribution disappears. For even greater post-etching times (Fig. 4b), a blue shift of the emission is appreciated.

In order to characterize the process with transmission electron microscopy (TEM), very thin freestanding layers $(500 \mathrm{~nm})$ were also produced with the same anodization currents as the previous samples. They were left in the electrolyte for different times (0, 1200 and $2400 \mathrm{~s})$, and the TEM images are shown in Fig. 5. A blurred pattern can be appreciated due to the roughness of the pores, taking into account that the width of the pores is much smaller than the total thickness of the sample. However, the images allow appreciating the porosity increase as an effect of post-etching.
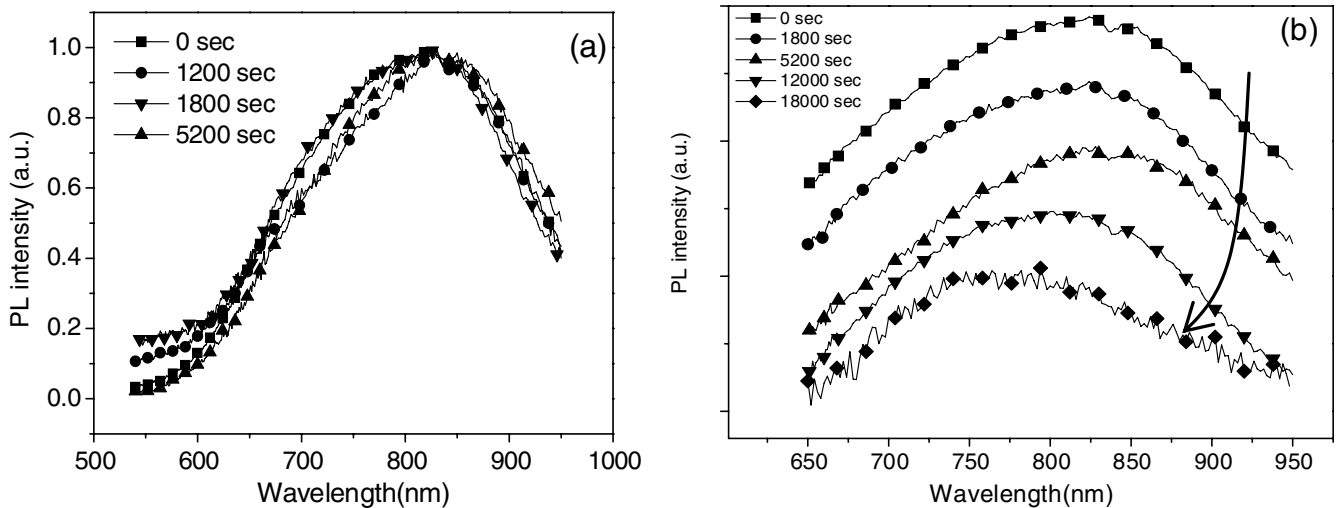

Fig. 4 (a) Normalized PL spectra for short post-etching time samples. (b) Waterfall plot of PL curves for long post-etching time samples. The arrow is a guide for the eye. 

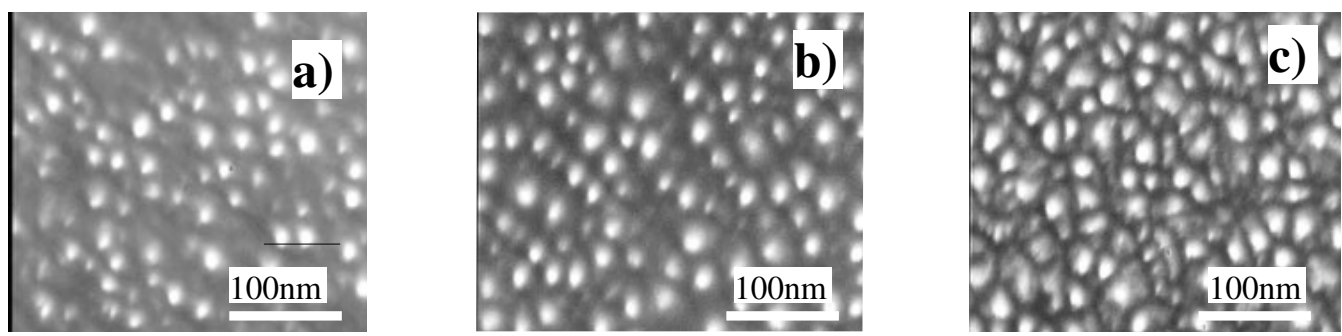

Fig. 5 TEM micrographs of a) a non post-etched sample, b) $1200 \mathrm{~s}$ and c) $2400 \mathrm{~s}$ of post-etching samples.

\section{Discussion and conclusions}

From the experimental study we have found that:

1. During the post-etching the refractive index of PS decreases following a slow chemical etching.

2. The rate of the decrease of the refractive index is characterized by two time scales: at short times a fast decrease in $n_{\mathrm{PS}}$ is observed and at long times a slower decrease in $n_{\mathrm{PS}}$ is observed;

3. A contribution at short wavelength in the PL emission appears and disappears at short times;

4. The maximum of the luminescence spectrum blueshifts for longer post-etching times.

5. Pore enlargement is clearly seen in TEM micrographs as the post-etching time increases.

We suggest a model that could explain the observed behaviors in PL as well as the in situ monitoring of the post-etching. It is based on the very inhomogeneous structure of the PS. It is well known that PS formed on $\mathrm{p}^{+}$-type silicon is mainly composed by a large $(3-10 \mathrm{~nm}$ in size) highly filamentary crystalline silicon network. These filaments are coated by smaller ( $1-4 \mathrm{~nm}$ in size) silicon nanostructures. These two different kinds of silicon nanostructures emit light in two different wavelength ranges because of the different quantum confinement characteristics. In addition, both structures are chemically etched by HF at different rates because of the different surface curvature and surface-volume ratio. Considering the inhomogeneous nature of PS we could explain our experimental observations as follows. The residence of the PS layer in the electrolyte causes a slow chemical etching of the structure with a negligible thickness layer modification but a significant decrease of the refractive index of the PS layer caused by the porosity change. The first fast exponential decrease of the refractive index is due to the etching of the small silicon nanocrystals. At the same time the short wavelength component of the luminescence emission, caused by the higher quantum confinement, increases and disappears. At longer post-etching times, a second process becomes dominant, characterized by a different rate. This process is the dissolution of the large filamentary silicon skeleton. The dissolution rate is now slower due to the smaller surfacevolume ratio and the larger surface curvature* radii. The luminescence emission band shifts to the blue following the progressive thinning of the filamentary silicon nanostructures, as observed in TEM images. In conclusion, we have demonstrated that chemical etching of PS samples occurs via a partial dissolution of silicon skeleton. This effect can be used to further tune the optical properties of PS.

Acknowledgements This work has been supported by the Science and Technology Ministry of Spain (MCYT) (Project No. MAT 2002-00044), the Canary Islands Government (Project No. PI2001/093, PI2001/074) and the European project EC-SINERGIA. We thank I. Martín and S. Gialanella for their contributions. C. O. acknowledges a fellowship granted by Cajacanarias and University of La Laguna.

\section{References}

[1] L. T. Canham, Appl. Phys. Lett. 57(10), 1046 (1990).

[2] A. Halimaoui, C. Oules, G. Bomchil, A. Bsiesy, F. Gaspard, R. Herino et al., Appl. Phys. Lett. 59, 304 (1991).

[3] M. J. Sailor, in: Properties of Porous Silicon, edited by L. T. Canham (IEE Inspec, London, U.K., 1997), p. 364.

[4] W. Theiß, Surf. Sci. Rep. 29(3/4), 91 (1997). 
[5] M. V. Wolkin, S. Chan, and P. M. Fauchet, phys. stat. sol. (a) 182, 573 (2000).

[6] W. Liu et al., J. Vac. Sci. Technol. B 21, 168 (2003).

[7] M. G. Berger, R. Arens-Fischer, M. Thonisson, M. Kruger, S. Billat et al., Thin Solid Films 297, 137 (1997).

[8] V. Agarwal and J. A. del Rio, Appl. Phys. Lett. 82, 1512 (2003).

[9] L. Pavesi, Riv. Nuovo Cimento 20, 1 (1997).

[10] S. Nagata, C. Domoto, T. Nishimura, and K. Iwameji, Appl. Phys. Lett. 72, 2945 (1998).

[11] M. B. Robinson, A. C. Dillon, and S. M. George, Appl. Phys. Lett. 62(13), 1493 (1993).

[12] M. Thönissen, M. G. Berger, S. Billat et al., Thin Solid Films 297, 92 (1997).

[13] Z. Gaburro, C. Oton, P. Bettotti, L. Dal Negro, G. Vijaya Prakash et al., J. Electrochem. Soc. 150, C 381 (2003). 